\title{
Ordering the oriented unicyclic graphs whose skew-spectral radius is bounded by 2
}

\author{
Ping-Feng Chen ${ }^{1}$, Guang-Hui Xu ${ }^{2 *}$ and Li-Pu Zhang ${ }^{2}$
}

\section{"Correspondence:}

ghxu@zafu.edu.cn

${ }^{2}$ School of Science, Zhejiang A\&F

University, Hangzhou, 311300,

China

Full list of author information is

available at the end of the article

\begin{abstract}
Let $S\left(G^{\sigma}\right)$ be the skew-adjacency matrix of an oriented graph $G^{\sigma}$ with $n$ vertices, and let $\lambda_{1}, \lambda_{2}, \ldots, \lambda_{n}$ be all eigenvalues of $S\left(G^{\sigma}\right)$. The skew-spectral radius $\rho_{s}\left(G^{\sigma}\right)$ of $G^{\sigma}$ is defined as $\max \left\{\left|\lambda_{1}\right|,\left|\lambda_{2}\right|, \ldots,\left|\lambda_{n}\right|\right\}$. A connected graph, in which the number of edges equals the number of vertices, is called a unicyclic graph. In this paper, the structure of oriented unicyclic graphs whose skew-spectral radius does not exceed 2 is investigated. We order all the oriented unicyclic graphs with $n$ vertices whose skew-spectral radius is bounded by 2.
\end{abstract}

MSC: $05 \mathrm{C} 50 ; 15 \mathrm{~A} 18$

Keywords: oriented unicyclic graph; skew-adjacency matrix; skew-spectral radius

\section{Introduction}

Let $G$ be a simple graph with $n$ vertices. The adjacency matrix $A=A(G)$ is the symmetric matrix $\left[a_{i j}\right]_{n \times n}$, where $a_{i j}=a_{j i}=1$ if $v_{i} v_{j}$ is an edge of $G$, otherwise, $a_{i j}=a_{j i}=0$. We call $\operatorname{det}(\lambda I-A)$ the characteristic polynomial of $G$, denoted by $\phi(G ; \lambda)$ (or abbreviated to $\phi(G)$ ). Since $A$ is symmetric, its eigenvalues $\lambda_{1}(G), \lambda_{2}(G), \ldots, \lambda_{n}(G)$ are real, and we assume that $\lambda_{1}(G) \geq \lambda_{2}(G) \geq \cdots \geq \lambda_{n}(G)$. We call $\rho(G)=\lambda_{1}(G)$ the adjacency spectral radius of $G$.

The class of all graphs $G$ whose largest (adjacency) spectral radius is bounded by 2 has been completely determined by Smith; see, for example, [1, 2]. Later, Hoffman [3], Cvetković et al. [4] gave a nearly complete description of all graphs $G$ with $2<\rho(G)<$ $\sqrt{2+\sqrt{5}}(\approx 2.0582)$. Their description was completed by Brouwer and Neumaier [5]. Then Woo and Neumaier [6] investigated the structure of graphs $G$ with $\sqrt{2+\sqrt{5}}<$ $\lambda_{\max }(G)<\frac{3}{2} \sqrt{2}(\approx 2.1312)$, Wang et al. [7] investigated the structure of graphs whose largest eigenvalue is close to $\frac{3}{2} \sqrt{2}$.

Another interesting problem that arises in the context of graph eigenvalues is to order graphs in some class with respect to the spectral radius or least eigenvalue. In 2003, Guo [8] gave the first six unicyclic graphs of order $n$ with larger spectral radius. Belardo et $a l$. [9] ordered graphs with spectral radius in the interval $(2, \sqrt{2+\sqrt{5}})$. In the paper [10], the first five unicyclic graphs on order $n$ in terms of their smaller least eigenvalues were determined.

The graph obtained from a simple undirected graph by assigning an orientation to each of its edges is referred as the oriented graph. Let $G^{\sigma}$ be an oriented graph with vertex set $\left\{v_{1}, v_{2}, \ldots, v_{n}\right\}$ and edge set $E\left(G^{\sigma}\right)$. The skew-adjacency matrix $S=S\left(G^{\sigma}\right)=\left[s_{i j}\right]_{n \times n}$ related

\section{算 Springer}

@2013 Chen et al.; licensee Springer. This is an Open Access article distributed under the terms of the Creative Commons Attribution License (http://creativecommons.org/licenses/by/2.0), which permits unrestricted use, distribution, and reproduction in any medium, provided the original work is properly cited. 
to $G^{\sigma}$ is defined as

$$
s_{i j}= \begin{cases}\stackrel{\circ}{1}, & \text { if there exists an edge with tail } v_{i} \text { and head } v_{j} ; \\ -\stackrel{i}{\mathbb{1}}, & \text { if there exists an edge with head } v_{i} \text { and tail } v_{j} ; \\ 0, & \text { otherwise, }\end{cases}
$$

where $\stackrel{i}{1}=\sqrt{-1}$ (note that the definition is slightly different from the one of the normal skew-adjacency matrix given by Adiga et al. [11]). Since $S\left(G^{\sigma}\right)$ is an Hermitian matrix, the eigenvalues $\lambda_{1}\left(G^{\sigma}\right), \lambda_{2}\left(G^{\sigma}\right), \ldots, \lambda_{n}\left(G^{\sigma}\right)$ of $S\left(G^{\sigma}\right)$ are all real numbers and, thus, can be arranged non-increase as

$$
\lambda_{1}\left(G^{\sigma}\right) \geq \lambda_{2}\left(G^{\sigma}\right) \geq \cdots \geq \lambda_{n}\left(G^{\sigma}\right) .
$$

The skew-spectral radius and the skew-characteristic polynomial of $G^{\sigma}$ are defined respectively as

$$
\rho_{s}\left(G^{\sigma}\right)=\max \left\{\left|\lambda_{1}\left(G^{\sigma}\right)\right|,\left|\lambda_{2}\left(G^{\sigma}\right)\right|, \ldots,\left|\lambda_{n}\left(G^{\sigma}\right)\right|\right\}
$$

and

$$
\phi\left(G^{\sigma} ; \lambda\right)=\operatorname{det}\left(\lambda I_{n}-S\left(G^{\sigma}\right)\right)
$$

Recently, much attention has been devoted to the skew-adjacency matrix of an oriented graph. In 2009, Shader and So [12] investigated the spectra of the skew-adjacency matrix of an oriented graph. In 2010, Adiga et al. [11] discussed the properties of the skew-energy of an oriented graph. In papers $[13,14]$, all the coefficients of the skew-characteristic polynomial of $G^{\sigma}$ in terms of $G$ were interpreted. Cavers et al. [15] discussed the graphs whose skew-adjacency matrices are all cospectral, and the relations between the matchings polynomial of a graph and the characteristic polynomials of its adjacency and skew-adjacency matrices. In [16], the author established a relation between $\rho_{s}\left(G^{\sigma}\right)$ and $\rho(G)$. Also, the author gave some results on the skew-spectral radii of $G^{\sigma}$ and its oriented subgraphs.

A connected graph in which the number of edges equals the number of vertices is called a unicyclic graph. In this paper, we will investigate the skew-spectral radius of an oriented unicyclic graph. The rest of this paper is organized as follows: In Section 2, we introduce some notations and preliminary results. In Section 3, all the oriented unicyclic graphs whose skew-spectral radius does not exceed 2 are determined. The result tells us that there is a big difference between the (adjacency) spectral radius of an undirected graph and the skew-spectral radius of its corresponding oriented graph. Furthermore, we order all the oriented unicyclic graphs with $n$ vertices whose skew-spectral radius is bounded by 2 in Section 4 .

\section{Preliminaries}

Let $G=(V, E)$ be a simple graph with vertex set $V=V(G)=\left\{v_{1}, v_{2}, \ldots, v_{n}\right\}$ and $e \in E(G)$. Denote by $G-e$ the graph obtained from $G$ by deleting the edge $e$ and by $G-v$ the graph obtained from $G$ by removing the vertex $v$ together with all edges incident to it. For a nonempty subset $W$ of $V(G)$, the subgraph with vertex set $W$ and edge set consisting of 
those pairs of vertices that are edges in $G$ is called an induced subgraph of $G$. Denote by $C_{n}, K_{1, n-1}$ and $P_{n}$ the cycle, the star and the path on $n$ vertices, respectively. Certainly, each subgraph of an oriented graph is also referred as an oriented graph and preserves the orientation of each edge.

Recall that the skew-adjacency matrix $S\left(G^{\sigma}\right)$ of any oriented graph $G^{\sigma}$ is Hermitian, then the well-known interlacing theorem for Hermitian matrices applies equally well to oriented graphs; see, for example, Theorem 4.3.8 of [17].

Lemma 2.1 Let $G^{\sigma}$ be an arbitrary oriented graph on $n$ vertices and $V^{\prime} \subseteq V(G)$. Suppose that $\left|V^{\prime}\right|=k$. Then

$$
\lambda_{i}\left(G^{\sigma}\right) \geq \lambda_{i}\left(G^{\sigma}-V^{\prime}\right) \geq \lambda_{i+k}\left(G^{\sigma}\right) \text { for } i=1,2, \ldots, n-k .
$$

Let $G^{\sigma}$ be an oriented graph, and let $C=v_{1} v_{2} \cdots v_{k} v_{1}(k \geq 3)$ be a cycle of $G$, where $v_{j}$ adjacent to $v_{j+1}$ for $j=1,2, \ldots, k-1$ and $v_{1}$ adjacent to $v_{k}$. Let also $S\left(G^{\sigma}\right)=\left[s_{i j}\right]_{n \times n}$ be the skew-adjacency matrix of $G^{\sigma}$ whose first $k$ rows and columns correspond to the vertices $v_{1}, v_{2}, \ldots, v_{k}$. The sign of the cycle $C^{\sigma}$, denoted by $\operatorname{sgn}\left(C^{\sigma}\right)$, is defined by

$$
\operatorname{sgn}\left(C^{\sigma}\right)=s_{1,2} s_{2,3} \cdots s_{k-1, k} s_{k, 1} \text {. }
$$

Let $\bar{C}=v_{1} v_{k} \cdots v_{2} v_{1}$ be the cycle by inverting the order of the vertices along the cycle $C$. Then one can verify that

$$
\operatorname{sgn}\left(\bar{C}^{\sigma}\right)= \begin{cases}-\operatorname{sgn}\left(C^{\sigma}\right), & \text { if } k \text { is odd } \\ \operatorname{sgn}\left(C^{\sigma}\right), & \text { if } k \text { is even }\end{cases}
$$

Moreover, $\operatorname{sgn}\left(C^{\sigma}\right)$ is either 1 or -1 if the length of $C$ is even; and $\operatorname{sgn}\left(C^{\sigma}\right)$ is either $\mathbb{1}$ or $-\stackrel{\AA}{1}$ if the length of $C$ is odd. For an even cycle, we simply refer it as a positive cycle or a negative cycle according to its sign. A positive even cycle is also named as oriented uniformly by Hou et al. [14].

On the skew-spectral radius of an oriented graph, we have obtained the following results. They will be useful in the proofs of the main results of this paper.

Lemma 2.2 ([16, Theorem 2.1]) Let $G^{\sigma}$ be an arbitrary connected oriented graph. Denote by $\rho(G)$ the (adjacency) spectral radius of $G$. Then

$$
\rho_{s}\left(G^{\sigma}\right) \leq \rho(G)
$$

with equality if and only if $G$ is bipartite and each cycle of $G$ is a positive even cycle.

Lemma 2.3 ([16, Theorem 3.2]) Let $G^{\sigma}$ be a connected oriented graph. Suppose that each even cycle of $G$ is positive. Then

(a) $\rho_{s}\left(G^{\sigma}\right)>\rho_{s}\left(G^{\sigma}-u\right)$ for any $u \in G$;

(b) $\rho_{s}\left(G^{\sigma}\right)>\rho_{s}\left(G^{\sigma}-e\right)$ for any $e \in G$.

Lemma 2.4 ([14, Theorem 2.4], [16, Theorem 3.1]) Let $G^{\sigma}$ be an oriented graph, and let $\phi\left(G^{\sigma}, \lambda\right)$ be its skew-characteristic polynomial. Then

(a) $\phi\left(G^{\sigma}, \lambda\right)=\lambda \phi\left(G^{\sigma}-u, \lambda\right)-\sum_{v \in N(u)} \phi\left(G^{\sigma}-u-v, \lambda\right)-2 \sum_{u \in C} \operatorname{sgn}(C) \phi\left(G^{\sigma}-C, \lambda\right)$, 
where the first summation is over all the vertices in $N(u)$, and the second summation is over all even cycles of $G$ containing the vertex $u$,

$$
\text { (b) } \phi\left(G^{\sigma}, \lambda\right)=\phi\left(G^{\sigma}-e, \lambda\right)-\phi\left(G^{\sigma}-u-v, \lambda\right)-2 \sum_{(u, v) \in C} \operatorname{sgn}(C) \phi\left(G^{\sigma}-C, \lambda\right) \text {, }
$$

where $e=(u, v)$ and the summation is over all even cycles of $G$ containing the edge $e$, and $\operatorname{sgn}(C)$ denotes the sign of the even cycle $C$.

Lemma 2.5 ([13, A part of Theorem 2.5]) Let $G^{\sigma}$ be an oriented graph, and let $\phi\left(G^{\sigma}, \lambda\right)$ be its skew-characteristic polynomial. Then

$$
\frac{d}{d \lambda} \phi\left(G^{\sigma}, \lambda\right)=\sum_{\nu \in V(G)} \phi\left(G^{\sigma}-v, \lambda\right)
$$

where $\frac{d}{d \lambda} \phi\left(G^{\sigma}, \lambda\right)$ denotes the derivative of $\phi\left(G^{\sigma}, \lambda\right)$.

Finally, we introduce a class of undirected graphs that will be often mentioned in this manuscript.

Denote by $P_{l_{1}, l_{2}, \ldots, l_{k}}$ a pathlike graph, which is defined as follows: we first draw $k(\geq 2)$ paths $P_{l_{1}}, P_{l_{2}}, \ldots, P_{l_{k}}$ of orders $l_{1}, l_{2}, \ldots, l_{k}$ respectively along a line and put two isolated vertices between each pair of those paths, then add edges between the two isolated vertices and the nearest end vertices of such a pair of paths such that the four newly added edges form a cycle $C_{4}$, where $l_{1}, l_{k} \geq 0$ and $l_{i} \geq 1$ for $i=2,3, \ldots, k-1$. Then $P_{l_{1}, l_{2}, \ldots, l_{k}}$ contains $\sum_{i=1}^{k} l_{i}+2 k-2$ vertices. Notice that if $l_{i}=1(i=2,3, \ldots, k-1)$, the two end vertices of the path $P_{l_{i}}$ are referred as overlap; if $l_{1}=0\left(l_{k}=0\right)$, the left (right) of the graph $P_{l_{1}, l_{2}, \ldots, l_{k}}$ has only two pendent vertices. Obviously, $P_{1,0}=K_{1,2}$, the star of order 3 , and $P_{1,1}=C_{4}$. In general, $P_{l_{1}, l_{2}}, P_{0, l_{1}, l_{2}}, P_{0, l_{1}, l_{2}, 0}$ are all unicyclic graphs containing $C_{4}$, where $l_{1}, l_{2} \geq 1$.

\section{The oriented unicyclic graphs whose skew-spectral radius does not exceed 2}

In this section, we determine all the oriented unicyclic graphs whose skew-spectral radius does not exceed 2 .

First, we introduce more notations. Denote by $T_{l_{1}, l_{2}, l_{3}}$ the starlike tree with exactly one vertex $v$ of degree 3 , and $T_{l_{1}, l_{2}, l_{3}}-v=P_{l_{1}} \cup P_{l_{2}} \cup P_{l_{3}}$, where $P_{l_{i}}$ is the path of order $l_{i}(i=$ $1,2,3)$.

Due to Smith, all undirected graphs whose (adjacency) spectral radius is bounded by 2 are completely determined as follows.

Lemma 3.1 ([2] or [1, Chapter 2.7.12]) All undirected graphs whose spectral radius does not exceed 2 are $C_{m}, P_{0, n-4,0}, T_{2,2,2}, T_{1,3,3}, T_{1,2,5}$ and their subgraphs, where $m \geq 3$ and $n \geq 5$.

By Lemma 2.4, to study the skew-spectrum properties of an oriented graph, we need only consider the sign of those even cycles. Moreover, Shader and So showed that $S\left(G^{\sigma}\right)$ has the same spectrum as that of its underlying tree for any oriented tree $G^{\sigma}$; see Theorem 2.5 of [12]. Consequently, combining with Lemma 2.2, the skew-spectral radius of each oriented graph whose underlying graph is as described in Lemma 3.1, regardless of the orientation of the oriented cycle $C_{n}^{\sigma}$, does not exceed 2. 
For convenience, we write:

$\mathcal{U}=\{G \mid G$ is a unicyclic graph $\}$.

$\mathcal{U}(m)=\left\{G \mid G\right.$ is a unicyclic graph in $\mathcal{U}$ containing the cycle $\left.C_{m}\right\}$.

$\mathcal{U}^{*}(m)=\left\{G \mid G\right.$ is a unicyclic graph in $\mathcal{U}(m)$ which is not the cycle $\left.C_{m}\right\}$.

$\mathcal{U}_{n}=\{G \mid G$ is a unicyclic graph on order $n\}$.

Moreover, let $C_{m}=v_{1} v_{2} \cdots v_{m} v_{1}$ be a cycle on $m$ vertices, and let $P_{l_{1}}, P_{l_{2}}, \ldots, P_{l_{m}}$ be $m$ paths with lengths $l_{1}, l_{2}, \ldots, l_{m}$ (perhaps some of them are empty), respectively. Denote by $C_{m}^{l_{1}, l_{2}, \ldots, l_{m}}$ the unicyclic undirected graph obtained from $C_{m}$ by joining $v_{i}$ to a pendent vertex of $P_{l_{i}}$ for $i=1,2, \ldots, m$. Suppose, without loss of generality, that $l_{1}=\max \left\{l_{i}: i=1,2, \ldots, m\right\}$, $l_{2} \geq l_{m}$, and write $C_{m}^{l_{1}, l_{2}, \ldots, l_{j}}$ instead of the standard $C_{m}^{l_{1}, l_{2}, \ldots, l_{j}, 0, \ldots, 0}$ if $l_{j+1}=l_{j+2}=\cdots=l_{m}=0$.

By Lemmas 2.2 and 2.4 or papers $[11,12]$, for a given unicyclic graph $G \in \mathcal{U}(m)$, we know that the skew-spectral radius of $G^{\sigma}$ is independent of its orientation if $m$ is odd. Therefore, we will briefly write $\vec{G}$ instead of the normal notation $G^{\sigma}$ if each cycle of $G$ is odd. If $m$ is even, then essentially, there exist two orientations $\sigma_{1}$ (the sign of the even cycle is positive) and $\sigma_{2}$ (the sign of the even cycle is negative) such that $\rho_{s}\left(G^{\sigma_{1}}\right)=\rho(G)$ and $\rho_{s}\left(G^{\sigma_{2}}\right)<\rho(G)$. Henceforth, we will briefly write $G^{-}$(or $G^{+}$) instead of $G^{\sigma}$ if the sign of each even cycle is negative (or positive). In particular, $G$ will also denote the oriented graph if $G$ is a tree since $\rho_{s}\left(G^{\sigma}\right)=\rho(G)$ in this case.

\subsection{The $C_{4}$-free oriented unicyclic graphs whose skew-spectral radius does not exceed 2}

Let $G^{\sigma}$ be an oriented graph with the property

$$
\rho_{s}\left(G^{\sigma}\right) \leq 2
$$

The property (3.1) is hereditary, because, as a direct consequence of Lemma 2.1 , for any induced subgraph $H \subset G, H^{\sigma}$ also satisfies (3.1). The inheritance (hereditary) of property (3.1) implies that there are minimal connected graphs that do not obey (3.1); such graphs are called forbidden subgraphs. It is easy to verify the following.

Lemma 3.2 Let $G \in \mathcal{U} \backslash \mathcal{U}(4)$ with $\rho_{s}\left(G^{\sigma}\right) \leq 2$. Then $\vec{C}_{3}^{3}, \vec{C}_{3}^{1,1}, \vec{C}_{3}(2), \vec{C}_{3}^{1}(2), \vec{C}_{5}^{1}, \vec{C}_{7}^{1}$ are forbidden, where $\vec{C}_{3}(2)$ (or $\vec{C}_{3}^{1}(2)$ ) denotes the oriented graph obtained by adding two pendent vertices to a vertex (or the pendent vertex) of $\vec{C}_{3}\left(\right.$ or $\left.\vec{C}_{3}^{1}\right)$.

Combining with Lemma 3.2 and the fact that $\rho_{s}(T)>2$ if the oriented tree $T$ contains an arbitrary tree described as Lemma 3.1 as a proper subgraph, we have the following result.

Theorem 3.1 Let $G \in \mathcal{U} \backslash \mathcal{U}(4)$ and $G \neq C_{m}$. Let also $\rho_{s}\left(G^{\sigma}\right) \leq 2$. Then $G^{\sigma}$ is one of $\vec{C}_{3}^{2}$, $\left(C_{6}^{2,0,0,2}\right)^{-},\left(C_{6}^{1,0,1,0,1}\right)^{-},\left(C_{8}^{1,0,0,0,1}\right)^{-}$and their induced oriented unicyclic subgraphs.

Proof Denote by $\operatorname{gir}(G)$ the girth of $G$. Let $\operatorname{gir}(G)=m$ and $C_{m}$ be the cycle of $G$ with vertex set $\left\{v_{1}, v_{2}, \ldots, v_{m}\right\}$ such that $v_{i}$ adjacent to $v_{i+1}$ for $i=1,2, \ldots, m-1$ and $v_{m}$ adjacent to $v_{1}$. (We should point out once again that in $C_{m}^{l_{1}, l_{2}, \ldots, l_{j}}(j \leq m)$, we always refer $v_{i}$ adjacent to one pendent vertex of $P_{l_{i}}$, a path with length $l_{i}$, for $i=1,2, \ldots, j$.) We divide our proof into the following four claims.

Claim 1 If $\operatorname{gir}(G)=3$, then $G^{\sigma} \in\left\{\vec{C}_{3}^{1}, \vec{C}_{3}^{2}\right\}$. 
The result follows from Lemma 3.2 that $\vec{C}_{3}^{3}, \vec{C}_{3}^{1,1}$ and $\vec{C}_{3}(2), \vec{C}_{3}^{1}(2)$ are forbidden.

Claim 2 If $\operatorname{gir}(G) \neq 3$, then $\operatorname{gir}(G) \in\{6,8\}$. Moreover, each induced even cycle of $G^{\sigma}$ is negative.

Let $\operatorname{gir}(G)=m$. Notice that $G$ is $C_{4}$-free, then $m \geq 5$ if $m \neq 3$, and, thus, $G$ contains the induced subgraph $C_{m}^{1}$ as $G \neq C_{n}$. From Lemma 3.2, both $\vec{C}_{5}^{1}$ and $\vec{C}_{7}^{1}$ are forbidden, thus, $m \neq 5,7$. Moreover, the graph obtained from $C_{m}^{1}$ by deleting the vertex $v_{5}$ is the tree $T_{1,3, m-5}$ for $m \geq 6$. Thus, there is an induced subgraph $T_{1,3,4}$ if $\operatorname{gir}(G) \geq 9$, which is a contradiction to Lemma 3.1. Hence, the former follows.

Assume to the contrary that there exists a positive even cycle $C_{m}^{+}$, then by Lemma 2.3, $\rho_{s}\left(G^{\sigma}\right) \geq \rho_{s}\left(\left(C_{m}^{1}\right)^{+}\right)>\rho_{s}\left(C_{m}^{+}\right)=2$, a contradiction. Thus, the latter follows.

Claim 3 If $\operatorname{gir}(G)=6$, then $G^{\sigma}$ is one of $\left(C_{6}^{1,0,1,0,1}\right)^{-},\left(C_{6}^{2,0,0,2}\right)^{-}$or their induced subgraphs.

By Claim 2, we always suppose that each cycle $\widehat{C}_{6}$ is negative.

We first claim that $G$ is of $C_{6}^{l_{1}, l_{2}, l_{3}, l_{4}, l_{5}, l_{6}}$, that is, each pendent tree adjacent to $v_{i}$ of $C_{6}$ is a path for $i=1,2, \ldots, 6$. Otherwise, assume that the pendent tree adjacent to $v_{1}$ is not a path, then the resultant graph by deleting vertex $v_{3}$ of $G$ is a tree and contains the tree $P_{0, l, 0}$ as a proper induced subgraph, and, thus, $\rho_{s}\left(G^{\sigma}\right)>\rho_{s}\left(P_{0, l, 0}\right)=2$ combining with Lemmas 2.3 and 3.2, a contradiction. Moreover, we have $l_{1} \leq 2$. Otherwise, $G-v_{4}$ contains $T_{2,2,3}$ as an induced subgraph. Notice that both $C_{6}^{1,1}-v_{4}$ and $C_{6}^{2,0,1}-v_{5}$ are trees and contain $P_{0,2,0}$ as a proper induced subgraph, then $G$ may be $C_{6}^{1,0,1,0,1}$ and $C_{6}^{2,0,0,2}$. By calculation, we have $\rho_{s}\left(\left(C_{6}^{1,0,1,0,1}\right)^{-}\right)=2$ and $\rho_{s}\left(\left(C_{6}^{2,0,0,2}\right)^{-}\right)=2$. Thus, the result follows.

Claim 4 If $\operatorname{gir}(G)=8$, then $G^{\sigma}$ is one of $\left(C_{8}^{1,0,0,0,1}\right)^{-}$or its induced subgraphs.

By Claim 2, the cycle $C_{8}^{\sigma}$ of $G^{\sigma}$ is negative. Notice that $C_{8}^{2}-v_{5}=T_{2,3,3}, C_{8}^{1,1}-v_{5}=T_{2,2,3}$, $C_{8}^{1,0,1}-v_{5}=T_{2,2,3}, C_{8}^{1,0,0,1}-v_{5}=T_{2,2,3}$, each of them has skew-spectral radius greater than 2 . Then $G^{\sigma}$ may be $\left(C_{8}^{1,0,0,0,1}\right)^{-}$. By calculation, we have $\rho_{s}\left(\left(C_{8}^{1,0,0,0,1}\right)^{-}\right)=2$. Thus, the result follows.

\subsection{The oriented unicyclic graphs in $\mathcal{U}(4)$ whose skew-spectral radius does not exceed 2}

Now, we consider the oriented unicyclic graphs in $\mathcal{U}(4)$. First, we have the following.

Lemma 3.3 Let $l_{1}, l_{2} \geq 1$. Then

(a) $\rho_{s}\left(P_{l_{1}, l_{2}}^{-}\right)<2$;

(b) $\rho_{s}\left(P_{0, l_{1}, l_{2}}^{-}\right)=\rho_{s}\left(P_{0, l_{1}, l_{2}, 0}^{-}\right)=2$.

Proof (a) Let $n=l_{1}+l_{2}+2$. We first show by induction on $n$ that

$$
\phi\left(P_{l_{1}, l_{2}}^{-}, 2\right)=4
$$

Let $l_{1} \geq l_{2}$. Then there is exactly one pathlike graph if $n=4$, namely, $P_{1,1}=C_{4}$. By calculation, we have

$$
\phi\left(P_{1,1}^{-}, 2\right)=4
$$


Suppose now that $n \geq 5$, and the result is true for the order no more than $n-1$. Applying Lemma 2.4 to the left pendent vertex of $P_{l_{1}, l_{2}}^{-}$, we have

$$
\phi\left(P_{l_{1}, l_{2}}^{-}, \lambda\right)=\lambda \phi\left(P_{l_{1}-1, l_{2}}^{-}, \lambda\right)-\phi\left(P_{l_{1}-2, l_{2}}^{-}, \lambda\right) .
$$

Then $\phi\left(P_{l_{1}, l_{2}}^{-}, 2\right)=4$ by induction hypothesis, and, thus, the result follows.

Let now $v$ be a vertex with degree 2 in $C_{4}$ of $P_{l_{1}, l_{2}}$. Then $P_{l_{1}, l_{2}}-v=P_{n-1}$, a path of order $n-1$. Let $\lambda_{1} \geq \lambda_{2} \geq \cdots \geq \lambda_{n}$ and $\bar{\lambda}_{1} \geq \bar{\lambda}_{2} \geq \cdots \geq \bar{\lambda}_{n-1}$ be all eigenvalues of $P_{l_{1}, l_{2}}^{-}$and $P_{n-1}$, respectively. By Lemma 2.1 and the fact that $\bar{\lambda}_{1}<2$, we have $\lambda_{2} \leq \bar{\lambda}_{1}<2$. On the other hand, we have

$$
\phi\left(P_{l_{1}, l_{2}}^{-}, \lambda\right)=\prod_{i=1}^{n}\left(\lambda-\lambda_{i}\right) .
$$

Consequently, $\lambda_{1}<2$, and, thus, $\rho_{s}\left(P_{l_{1}, l_{2}}^{-}\right)<2$ by Eq. (3.2). Thus, the result (a) holds.

(b) We first show that 2 is an eigenvalue of $P_{0, l_{1}, l_{2}}^{-}$.

$$
\phi\left(P_{0, l_{1}, l_{2}}^{-}, \lambda\right)=\lambda \phi\left(P_{l_{1}+1, l_{2}}^{-}, \lambda\right)-\lambda \phi\left(P_{l_{1}-1, l_{2}}^{-}, \lambda\right) .
$$

By the proof of the result (a), we know that

$$
\phi\left(P_{l_{1}+1, l_{2}}^{-}, 2\right)=\phi\left(P_{l_{1}-1, l_{2}}^{-}, 2\right)=4 .
$$

It tells us that

$$
\phi\left(P_{0, l_{1}+1, l_{2}}^{-}, 2\right)=0 .
$$

Note that $\lambda_{2}\left(P_{0, l_{1}+1, l_{2}}^{-}\right)<2$. We know that $\rho_{s}\left(P_{0, l_{1}, l_{2}}^{-}\right)=2$.

Now, we show that 2 is also an eigenvalue of $P_{0, l_{1}, l_{2}, 0}^{-}$. Applying Lemma 2.5, we have

$$
\frac{d}{d \lambda} \phi\left(P_{0, l_{1}, l_{2}, 0}^{-}, \lambda\right)=\sum_{v} \phi\left(P_{0, l_{1}, l_{2}, 0}^{-}-v, \lambda\right)
$$

It is easy to see that 2 is an eigenvalue of each oriented graph $P_{0, l_{1}, l_{2}, 0}^{-}-v$. Thus, 2 is an eigenvalue of $P_{0, l_{1}, l_{2}, 0}^{-}$with multiplicity 2 .

By calculation, we have the following.

Lemma 3.4 Let $G \in \mathcal{U}(4)$ with $\rho_{s}\left(G^{\sigma}\right) \leq 2$. Then $G_{i}^{-}(i=1,2, \ldots, 7)$ are forbidden, where $G_{1}=C_{4}^{5,1}, G_{2}=C_{4}^{3,2}, G_{3}=C_{4}^{4,1,1}, G_{4}=C_{4}^{3,1,2}, G_{5}=C_{4}^{2,2,1}, G_{6}=C_{4}^{3,1,0,1}$ and $G_{7}=P_{0, l_{1}, l_{2}, 0}^{1}$, which denotes the graph obtained by adding a pendent vertex to a vertex of $P_{0, l_{1}, l_{2}, 0}$.

Combining with Lemma 3.4 and the fact that $\rho_{s}(T)>2$ if the oriented tree $T$ contains an arbitrary tree described as Lemma 3.1 as a proper subgraph, we have the following result.

Theorem 3.2 Let $G \in \mathcal{U}^{*}(4)$ and $\rho_{s}\left(G^{\sigma}\right) \leq 2$. Then $G^{\sigma}$ is one of $\left(C_{4}^{4,1}\right)^{-},\left(C_{4}^{3,1,1}\right)^{-},\left(C_{4}^{2,1,2,1}\right)^{-}$, $\left(C_{4}^{2,2}\right)^{-}$and $P_{0, l_{1}, l_{2}, 0}^{-}$or their induced oriented unicyclic subgraphs. 
Proof Note that the induced cycle $C_{4}^{\sigma}$ of $G^{\sigma}$ must be negative. By Lemma 3.3, we can assume that $G \neq P_{0, l_{1}, l_{2}, 0}$.

Case 1. $G \neq C_{4}^{l_{1}, l_{2}, l_{3}, l_{4}}$.

Then $G$ contains an induced tree $T$ such that $T$ has a proper induced subgraph $P_{0, l, 0}$. It means that $\rho_{s}\left(G^{\sigma}\right)>\rho\left(P_{0, l, 0}\right)=2$, a contradiction.

Case 2. $G=C_{4}^{l_{1}, l_{2}, l_{3}, l_{4}}$.

Then, by Lemma 3.4, we know that $l_{1} \leq 4$ and $l_{2} \geq 1$. Thus, it is not difficult to see that the possible oriented graphs are $\left(C_{4}^{4,1}\right)^{-},\left(C_{4}^{3,1,1}\right)^{-},\left(C_{4}^{2,1,2,1}\right)^{-},\left(C_{4}^{2,2}\right)^{-}$or their induced oriented unicyclic subgraphs by Lemma 3.4. Moreover, taking some computations, we know the skew-spectral radius of each above oriented graph does not exceed 2.

Combining with Lemma 3.3, the result follows.

\subsection{The oriented unicyclic graphs whose skew-spectral radius does not exceed 2}

Putting Lemma 3.1 together with Theorem 3.1 and Theorem 3.2, we have the following.

Theorem 3.3 Let $G \in \mathcal{U}$ and $\rho_{s}\left(G^{\sigma}\right) \leq 2$. Then $G^{\sigma}$ is one of $C_{m}^{\sigma}, \vec{C}_{3}^{2},\left(C_{4}^{4,1}\right)^{-},\left(C_{4}^{3,1,1}\right)^{-}$, $\left(C_{4}^{2,1,2,1}\right)^{-},\left(C_{4}^{2,2}\right)^{-},\left(C_{6}^{2,0,0,2}\right)^{-},\left(C_{6}^{1,0,1,0,1}\right)^{-},\left(C_{8}^{1,0,0,0,1}\right)^{-}$and $P_{0, l_{1}, l_{2}, 0}^{-}$or their induced oriented unicyclic subgraphs, where the orientation of $C_{m}^{\sigma}$ is arbitrary.

Moreover, by calculation, we have the following two corollaries from Theorem 3.3.

Corollary 3.1 Let $G \in \mathcal{U}$ and $\rho_{s}\left(G^{\sigma}\right)=2$. Then $G^{\sigma}$ is one of the following oriented graphs.

(a) $C_{m}^{+}$, where $m$ is even;

(b) $P_{0, l_{1}, l_{2}}^{-}, P_{0, l_{1}, l_{2}, 0}^{-}$, where $l_{1}, l_{2} \geq 1$;

(c) $\vec{C}_{3}^{2},\left(C_{4}^{4,1}\right)^{-},\left(C_{4}^{3,1,1}\right)^{-},\left(C_{4}^{2,1,2,1}\right)^{-},\left(C_{4}^{2,1,2}\right)^{-},\left(C_{4}^{2,1,1,1}\right)^{-},\left(C_{4}^{2,1,0,1}\right)^{-},\left(C_{4}^{2,2}\right)^{-},\left(C_{6}^{2,0,0,2}\right)^{-}$, $\left(C_{6}^{1,0,1,0,1}\right)^{-},\left(C_{8}^{1,0,0,0,1}\right)^{-}$and $\left(C_{8}^{1}\right)^{-}$.

Corollary 3.2 Let $G \in \mathcal{U}$ and $\rho_{s}\left(G^{\sigma}\right)<2$. Then $G^{\sigma}$ is one of the following oriented graphs or their induced oriented unicyclic subgraphs.

(a) $C_{m}^{\sigma}$, where $m$ is odd, or $m$ is even, and the sign of $C_{m}^{\sigma}$ is negative;

(b) $P_{l_{1}, l_{2}}^{-}$, where $l_{1}, l_{2} \geq 1$;

(c) $\vec{C}_{3}^{1},\left(C_{4}^{3,1}\right)^{-},\left(C_{4}^{2,1,1}\right)^{-},\left(C_{4}^{1,1,1,1}\right)^{-},\left(C_{6}^{2,0,0,1}\right)^{-},\left(C_{6}^{1,0,1}\right)^{-}$.

\section{Ordering the oriented unicyclic graphs whose skew-spectral radius is bounded by 2}

In this section, we discuss the skew-spectral radii of oriented unicyclic graphs in $\mathcal{U}_{n}$. Let $G \in \mathcal{U}_{n}$ and $\rho_{s}\left(G^{\sigma}\right)<2$. By Corollary 3.2, we know that $G^{\sigma}$ is $C_{n}^{\sigma}$ (where $n$ is odd, or $n$ is even, and the sign is negative) or $P_{l, n-l}^{-}$(where $l \geq 1$ ) if $n \geq 10$. This makes it possible to order the oriented unicyclic graphs whose skew-spectral radius is bounded by 2 .

Lemma 4.1 Let $l_{2} \geq l_{1} \geq 2$. Then $\rho_{s}\left(P_{l_{1}, l_{2}}^{-}\right)<\rho_{s}\left(P_{l_{1}-1, l_{2}+1}^{-}\right)$.

Proof By Lemma 2.4, we have

$$
\begin{aligned}
& \phi\left(P_{l_{1}, l_{2}}^{-}\right)=\lambda \phi\left(P_{l_{1}+l_{2}+1}\right)-\phi\left(P_{l_{1}-1}\right) \phi\left(P_{l_{2}+1}\right)-\phi\left(P_{l_{1}+1}\right) \phi\left(P_{l_{2}-1}\right)+2 \phi\left(P_{l_{1}-1}\right) \phi\left(P_{l_{2}-1}\right) ; \\
& \phi\left(P_{l_{1}-1, l_{2}+1}^{-}\right)=\lambda \phi\left(P_{l_{1}+l_{2}+1}\right)-\phi\left(P_{l_{1}-2}\right) \phi\left(P_{l_{2}+2}\right)-\phi\left(P_{l_{1}}\right) \phi\left(P_{l_{2}}\right)+2 \phi\left(P_{l_{1}-2}\right) \phi\left(P_{l_{2}}\right) .
\end{aligned}
$$


Thus,

$$
\begin{aligned}
& \phi\left(P_{l_{1}, l_{2}}^{-}\right)-\phi\left(P_{l_{1}-1, l_{2}+1}^{-}\right) \\
& =\left[\phi\left(P_{l_{1}-2}\right) \phi\left(P_{l_{2}+2}\right)-\phi\left(P_{l_{1}-1}\right) \phi\left(P_{l_{2}+1}\right)\right]+\left[\phi\left(P_{l_{1}}\right) \phi\left(P_{l_{2}}\right)-\phi\left(P_{l_{1}+1}\right) \phi\left(P_{l_{2}-1}\right)\right] \\
& \quad+2\left[\phi\left(P_{l_{1}-1}\right) \phi\left(P_{l_{2}-1}\right)-\phi\left(P_{l_{1}-2}\right) \phi\left(P_{l_{2}}\right)\right] .
\end{aligned}
$$

Moreover, we have

$$
\begin{aligned}
\phi & \left(P_{l_{1}-2}\right) \phi\left(P_{l_{2}+2}\right)-\phi\left(P_{l_{1}-1}\right) \phi\left(P_{l_{2}+1}\right) \\
& =\phi\left(P_{l_{1}-2}\right)\left[\lambda \phi\left(P_{l_{2}+1}\right)-\phi\left(P_{l_{2}}\right)\right]-\phi\left(P_{l_{2}+1}\right)\left[\lambda \phi\left(P_{l_{1}-2}\right)-\phi\left(P_{l_{1}-3}\right)\right] \\
& =\phi\left(P_{l_{1}-3}\right) \phi\left(P_{l_{2}+1}\right)-\phi\left(P_{l_{1}-2}\right) \phi\left(P_{l_{2}}\right) \\
& =\phi\left(P_{0}\right) \phi\left(P_{l_{2}-l_{1}+4}\right)-\phi\left(P_{1}\right) \phi\left(P_{l_{2}-l_{1}+3}\right) \\
& =\phi\left(P_{l_{2}-l_{1}+4}\right)-\lambda \phi\left(P_{l_{2}-l_{1}+3}\right) \\
& =-\phi\left(P_{l_{2}-l_{1}+2}\right), \\
\phi & \left(P_{l_{1}}\right) \phi\left(P_{l_{2}}\right)-\phi\left(P_{l_{1}+1}\right) \phi\left(P_{l_{2}-1}\right) \\
& =\phi\left(P_{l_{1}}\right)\left[\lambda \phi\left(P_{l_{2}-1}\right)-\phi\left(P_{l_{2}-2}\right)\right]-\phi\left(P_{l_{2}-1}\right)\left[\lambda \phi\left(P_{l_{1}}\right)-\phi\left(P_{l_{1}-1}\right)\right] \\
& =\phi\left(P_{l_{1}-1}\right) \phi\left(P_{l_{2}-1}\right)-\phi\left(P_{l_{1}}\right) \phi\left(P_{l_{2}-2}\right) \\
& =\phi\left(P_{0}\right) \phi\left(P_{l_{2}-l_{1}}\right)-\phi\left(P_{1}\right) \phi\left(P_{l_{2}-l_{1}-1}\right) \\
& =\phi\left(P_{l_{2}-l_{1}}\right)-\lambda \phi\left(P_{l_{2}-l_{1}-1}\right) \\
& =-\phi\left(P_{l_{2}-l_{1}-2}\right),
\end{aligned}
$$

where $l_{2}-l_{1} \geq 2$. It is easy to know that

$$
\phi\left(P_{l_{1}}\right) \phi\left(P_{l_{2}}\right)-\phi\left(P_{l_{1}+1}\right) \phi\left(P_{l_{2}-1}\right)=1 \quad \text { if } l_{2}-l_{1}=0
$$

and

$$
\phi\left(P_{l_{1}}\right) \phi\left(P_{l_{2}}\right)-\phi\left(P_{l_{1}+1}\right) \phi\left(P_{l_{2}-1}\right)=0 \quad \text { if } l_{2}-l_{1}=1 .
$$

Similarly, we have

$$
\begin{aligned}
\phi & \left(P_{l_{1}-1}\right) \phi\left(P_{l_{2}-1}\right)-\phi\left(P_{l_{1}-2}\right) \phi\left(P_{l_{2}}\right) \\
& =\phi\left(P_{l_{2}-1}\right)\left[\lambda \phi\left(P_{l_{1}-2}\right)-\phi\left(P_{l_{1}-3}\right)\right]-\phi\left(P_{l_{1}-2}\right)\left[\lambda \phi\left(P_{l_{2}-1}\right)-\phi\left(P_{l_{2}-2}\right)\right] \\
& =\phi\left(P_{l_{1}-2}\right) \phi\left(P_{l_{2}-2}\right)-\phi\left(P_{l_{1}-3}\right) \phi\left(P_{l_{2}-1}\right) \\
& =\phi\left(P_{1}\right) \phi\left(P_{l_{2}-l_{1}+1}\right)-\phi\left(P_{0}\right) \phi\left(P_{l_{2}-l_{1}+2}\right) \\
& =\lambda \phi\left(P_{l_{2}-l_{1}}\right)-\phi\left(P_{l_{2}-l_{1}+1}\right) \\
& =\phi\left(P_{l_{2}-l_{1}}\right) .
\end{aligned}
$$

Hence,

$$
\phi\left(P_{l_{1}, l_{2}}^{-}\right)-\phi\left(P_{l_{1}-1, l_{2}+1}^{-}\right)=-\phi\left(P_{l_{2}-l_{1}+2}\right)-\phi\left(P_{l_{2}-l_{1}-2}\right)+2 \phi\left(P_{l_{2}-l_{1}}\right) .
$$


Let $l_{2}-l_{1}=k$. Then for $k \geq 2$, we have

$$
\begin{aligned}
\phi\left(P_{l_{1}, l_{2}}^{-}\right)-\phi\left(P_{l_{1}-1, l_{2}+1}^{-}\right) & =-\phi\left(P_{k+2}\right)-\phi\left(P_{k-2}\right)+2 \phi\left(P_{k}\right) \\
& =-\left[\phi\left(P_{2}\right) \phi\left(P_{k}\right)-\phi\left(P_{1}\right) \phi\left(P_{k-1}\right)\right]-\phi\left(P_{k-2}\right)+2 \phi\left(P_{k}\right) \\
& =\left(3-\lambda^{2}\right) \phi\left(P_{k}\right)+\phi\left(P_{1}\right) \phi\left(P_{k-1}\right)-\phi\left(P_{k-2}\right) \\
& =\left(4-\lambda^{2}\right) \phi\left(P_{k}\right) .
\end{aligned}
$$

Obviously, the above equality also holds for $k=0,1$. It means that $\phi\left(P_{l_{1}, l_{2}}^{-}, \rho_{s}\left(P_{l_{1}-1, l_{2}+1}^{-}\right)\right)>$ 0 , since $\rho_{s}\left(P_{l_{1}-1, l_{2}+1}^{-}\right)<2$. Thus, $\rho_{s}\left(P_{l_{1}, l_{2}}^{-}\right)<\rho_{s}\left(P_{l_{1}-1, l_{2}+1}^{-}\right)$.

By Lemma 4.1, we know that

$$
\rho_{s}\left(P_{\left\lfloor\frac{n-2}{2}\right\rfloor,\left\lceil\frac{n-2}{2}\right\rceil}^{-}\right)<\cdots<\rho_{s}\left(P_{2, n-4}^{-}\right)<\rho_{s}\left(P_{1, n-3}^{-}\right) .
$$

Now, we need only to compare the skew-spectral radii of $P_{l_{1}, l_{2}}^{-}$and $C_{n}^{\sigma}$. In fact, we have the following.

Lemma 4.2 Let $n \geq 4$. Then we have

(a) $\rho_{s}\left(P_{1, n-3}^{-}\right)<\rho_{s}\left(\vec{C}_{n}\right)$ if $n$ is odd;

(b) $\rho_{s}\left(P_{\frac{n-2}{2}, \frac{n-2}{2}}^{-}\right)=\rho_{s}\left(C_{n}^{-}\right)$if $n$ is even.

Proof Note that by paper [11]

$$
\rho_{s}\left(C_{n}^{\sigma}\right)= \begin{cases}2 \cos \frac{\pi}{2 n}, & \text { if } n \text { is odd; } \\ 2 \cos \frac{\pi}{n}, & \text { if } n \text { is even and the sign of the cycle is negative. }\end{cases}
$$

Moreover, we have $\rho_{s}\left(P_{0, n-2}\right)=2 \cos \frac{\pi}{2 n-2}$. Thus, $\rho_{s}\left(\vec{C}_{n}\right)>\rho_{s}\left(P_{0, n-2}\right)$ if $n$ is odd.

On the other hand, we have

$$
\begin{aligned}
& \phi\left(P_{1, n-3}^{-}\right)=\lambda \phi\left(P_{n-1}\right)-\phi\left(P_{n-2}\right)-\phi\left(P_{2}\right) \phi\left(P_{n-4}\right)+2 \phi\left(P_{n-4}\right) ; \\
& \phi\left(P_{0, n-2}\right)=\lambda \phi\left(P_{n-1}\right)-\lambda \phi\left(P_{n-3}\right) .
\end{aligned}
$$

Thus,

$$
\begin{aligned}
\phi\left(P_{1, n-3}^{-}\right)-\phi\left(P_{0, n-2}\right) & =-\phi\left(P_{2}\right) \phi\left(P_{n-4}\right)+2 \phi\left(P_{n-4}\right) \\
& =\left(4-\lambda^{2}\right) \phi\left(P_{n-4}\right) .
\end{aligned}
$$

It means that $\rho_{s}\left(P_{1, n-3}^{-}\right)<\rho_{s}\left(P_{0, n-2}\right)$. Then the result (a) follows.

If $n$ is even, then let $l=\frac{n-2}{2}$. We have

$$
\begin{aligned}
\phi\left(P_{l, l}^{-}\right) & =\lambda \phi\left(P_{n-1}\right)-2 \phi\left(P_{l-1}\right) \phi\left(P_{l+1}\right)+2 \phi\left(P_{l-1}\right) \phi\left(P_{l-1}\right) ; \\
\phi\left(C_{n}^{-}\right) & =\lambda \phi\left(P_{n-1}\right)-2 \phi\left(P_{n-2}\right)+2 \\
& =\lambda \phi\left(P_{n-1}\right)-2 \phi\left(P_{l-1}\right) \phi\left(P_{l+1}\right)+2 \phi\left(P_{l-2}\right) \phi\left(P_{l}\right)+2 .
\end{aligned}
$$


Thus,

$$
\begin{aligned}
\phi\left(P_{l, l}^{-}\right)-\phi\left(C_{n}^{-}\right) & =-2 \phi\left(P_{l-2}\right) \phi\left(P_{l}\right)+2 \phi\left(P_{l-1}\right) \phi\left(P_{l-1}\right)-2 \\
& =2\left[\phi\left(P_{l-2}\right) \phi\left(P_{l-2}\right)-\phi\left(P_{l-3}\right) \phi\left(P_{l-1}\right)\right]-2 \\
& =2\left[\phi\left(P_{1}\right) \phi\left(P_{1}\right)-\phi\left(P_{0}\right) \phi\left(P_{2}\right)\right]-2 \\
& =0 .
\end{aligned}
$$

Then the result (b) holds.

By Lemmas 4.1 and 4.2, we obtain the following interesting result.

Theorem 4.1 Let $G^{\sigma}$ be an oriented unicyclic graph on order $n(n \geq 10) . G^{\sigma} \neq P_{l_{1}, l_{2}}^{-}, C_{n}^{\sigma}$, where $n=l_{1}+l_{2}+2$ and $C_{n}^{\sigma}=C_{n}^{-}$if $n$ is even. Then

(a) $\rho_{s}\left(P_{\frac{n-3}{2}, \frac{n-1}{2}}^{-}\right)<\cdots<\rho_{s}\left(P_{1, n-3}^{-}\right)<\rho_{s}\left(\vec{C}_{n}\right)<2 \leq \rho_{s}\left(G^{\sigma}\right)$ if $n$ is odd;

(b) $\rho_{s}\left(C_{n}^{-}\right)=\rho_{s}\left(P_{\frac{n-2}{2}, \frac{n-2}{2}}^{-}\right)<\cdots<\rho_{s}\left(P_{1, n-3}^{-}\right)<2 \leq \rho_{s}\left(G^{\sigma}\right)$ if $n$ is even.

Combining with Corollary 3.1, we have ordered all the oriented unicyclic graphs with $n$ vertices whose skew-spectral radius is bounded by 2 .

\section{Competing interests}

The authors declare that they have no competing interests.

\section{Authors' contributions}

PC carried out the studies of the skew-spectral radii and drafted the manuscript. GX conceived of the study and finished the final manuscript. LZ participated in the design of the study and some calculation. All authors read and approved the final manuscript.

\section{Author details}

'School of Information Engineering, Zhejiang A\&F University, Hangzhou, 311300, China. ${ }^{2}$ School of Science, Zhejiang A\&F University, Hangzhou, 311300, China.

\section{Acknowledgements}

The authors are grateful to the referees for their valuable comments and suggestions, which led to a great improvement of the original manuscript. This work was supported by the National Natural Science Foundation of China (No. 11171373) the Zhejiang Provincial Natural Science Foundation of China (LY12A01016).

\section{Received: 24 March 2013 Accepted: 23 September 2013 Published: 07 Nov 2013}

\section{References}

1. Cvetković, D, Doob, M, Sachs, H: Spectra of Graphs. Academic Press, New York (1980)

2. Smith, JH: Some properties of the spectrum of a graph. In: Guy, R (ed.) Combinatorial Structures and Their Applications, pp. 403-406. Gordon \& Breach, New York (1970)

3. Hoffman, A: On limit points of spectral radii of non-negative symmetrical integral matrices. In: Graph Theory and Applications. Lecture Notes in Math., vol. 303, pp. 165-172 (1972)

4. Cvetković, D, Doob, M, Gutman, I: On graphs whose spectral radius does not exceed $\sqrt{2+\sqrt{5}}$. Ars Comb. 14, 225-239 (1982)

5. Brouwer, $A E$, Neumaier, $A$ : The graphs with largest eigenvalue between 2 and $\sqrt{2+\sqrt{5}}$. Linear Algebra Appl. $114 / 115,273-276(1989)$

6. Woo, R, Neumaier, A: On graphs whose spectral radius is bounded by $\frac{3}{2} \sqrt{2}$. Graphs Comb. $23,713-726$ (2007)

7. Wang, JF, Huang, QX, An, XH, Belardo, F: Some notes on graphs whose spectral radius is close to $\frac{3}{2} \sqrt{2}$. Linear Algebra Appl. 429, 1606-1618 (2008)

8. Guo, SG: The first six unicyclic graphs of order $n$ with larger spectral radius. Appl. Math. J. Chin. Univ. Ser. A 18(4) 480-486 (2003) (in Chinese)

9. Belardo, F, Li Marzi, EM, Simić, SK: Ordering graphs with index in the interval $(2, \sqrt{2+\sqrt{5}})$. Discrete Appl. Math. 156, 1670-1682 (2008)

10. Xu, GH: Ordering unicyclic graphs in terms of their smaller least eigenvalues. J. Inequal. Appl. 2010, Article ID 591758 (2010)

11. Adiga, C, Balakrishnan, R, So, WS: The skew energy of a graph. Linear Algebra Appl. 432, 1825-1835 (2010)

12. Shader, B, So, WS: Skew spectra of oriented graphs. Electron. J. Comb. 16, N32 (2009) 
13. Gong, SC, Xu, GH: The characteristic polynomial and the matchings polynomial of a weighted oriented graph. Linear Algebra Appl. 436, 465-471 (2012)

14. Hou, YP, Lei, TG: Characteristic polynomials of skew-adjacency matrices of oriented graphs. Electron. J. Comb. 18, P156 (2011)

15. Cavers, M, Cioabă, SM, Fallat, S, Gregory, DA, Haemerse, WH, Kirkland, SJ, McDonald, JJ, Tsatsomeros, M: Skew-adjacency matrices of graphs. Linear Algebra Appl. 436, 4512-4529 (2012)

16. Xu, GH: Some inequalities on the skew-spectral radii of oriented graphs. J. Inequal. Appl. 2012, 211 (2012)

17. Horn, R, Johnson, C: Matrix Analysis. Cambridge University Press, Cambridge (1989)

10.1186/1029-242X-2013-495

Cite this article as: Chen et al.: Ordering the oriented unicyclic graphs whose skew-spectral radius is bounded by 2. Journal of Inequalities and Applications 2013, 2013:495

Submit your manuscript to a SpringerOpen ${ }^{\odot}$ journal and benefit from:

- Convenient online submission

- Rigorous peer review

- Immediate publication on acceptance

- Open access: articles freely available online

- High visibility within the field

- Retaining the copyright to your article

Submit your next manuscript at $>$ springeropen.com 